\title{
Battery Grouping with Time Series Clustering Based on Affinity Propagation
}

\author{
Zhiwei He, Mingyu Gao *, Guojin Ma, Yuanyuan Liu and Lijun Tang \\ Department of Electronic \& Information, Hangzhou Dianzi University, 2nd Street, \\ Xiasha Higher Education Zone, Hangzhou 310018, China; zwhe@hdu.edu.cn (Z.H.); magj@hdu.edu.cn (G.M.); \\ liuyuanyuan@hdu.edu.cn (Y.L.); littlegoldfish1019@163.com (L.T.) \\ * Correspondence: mackgao@hdu.edu.cn; Tel./Fax: +86-571-8691-9153
}

Academic Editors: José C. Riquelme, Alicia Troncoso and Francisco Martínez-Álvarez Received: 11 April 2016; Accepted: 12 July 2016; Published: 19 July 2016

\begin{abstract}
Battery grouping is a technology widely used to improve the performance of battery packs. In this paper, we propose a time series clustering based battery grouping method. The proposed method utilizes the whole battery charge/discharge sequence for battery grouping. The time sequences are first denoised with a wavelet denoising technique. The similarity matrix is then computed with the dynamic time warping distance, and finally the time series are clustered with the affinity propagation algorithm according to the calculated similarity matrices. The silhouette index is utilized for assessing the performance of the proposed battery grouping method. Test results show that the proposed battery grouping method is effective.
\end{abstract}

Keywords: battery grouping; affinity propagation; time series clustering; wavelet denoising

\section{Introduction}

With the development of economics, people pay more and more attention to environmental protection, and new energy vehicles have been irresistible traffic tools for human beings. Power batteries, as the energy source of electric vehicles (EVs), play important roles in the whole EV system. However, the small capacity and low voltage of a single battery cell cannot meet the needs of an EV; thus people generally group many new battery cells together to form a battery pack to provide enough power for the EV [1]. Because of the differences in the characteristics, some of the batteries in a battery pack can easily be over-charged or over-discharged, or the potential of most batteries in a battery pack will not exhaust, which will affect the performance and service life of the whole battery pack. Moreover, the inconsistency of the batteries will make monitoring and management of the states, such as estimating the state of charge (SOC) $[2,3]$ and the state of health $(\mathrm{SOH})[4,5]$ of the battery pack, much more difficult. Therefore, it is very important to choose a suitable battery grouping method to assemble batteries with similar characteristics into a pack by a serial and/or parallel connection.

At present, there are three types of battery grouping methods as follows: single characteristic based battery grouping, multiple characteristics based battery grouping, and dynamic characteristics based battery grouping.

In general, the characteristics used for battery grouping include the battery terminal voltage, internal resistance, static capacity, self-discharge rate, etc. The single characteristic based battery grouping methods choose only one of these characteristics as the grouping criteria. For example, the cell capacity is usually used as the criteria for battery grouping, i.e., batteries with similar capacities are grouped together to form a battery pack. Internal resistance is another widely considered characteristic to build battery packs [6]. People may also consider thermal characteristics for building a battery pack [7]. Unfortunately, a single characteristic is generally too limited to present the performance 
of the battery; therefore, single characteristic based battery grouping methods commonly have poor performance.

Multiple characteristics based battery grouping methods combine several characteristics together as the grouping criteria. Multiple characteristics can better characterize the battery; thus, the performance of this grouping method is often greatly improved compared with single characteristic based grouping methods. For example, many battery manufactories in China combine the discharge open-circuit cutoff voltage and the charge static terminal voltage as the standard for battery grouping [8]. However, generally speaking, battery characteristics are often assessed when the battery is in a stable state without consideration of the dynamic performance of the battery; thus, even the combination of multiple characteristics still does not completely reflect the battery performance, especially the dynamic performance.

Dynamic characteristics reflect the working performance of a battery; thus, dynamic characteristics based battery grouping methods have the best performance compared to the other two methods. One of the widely applied battery dynamic characteristics is electrochemical impedance spectroscopy (EIS) [9], which has been used for battery SOC estimation [10], battery grouping [11], etc. The main problem of EIS is twofold. First, the EIS measurement needs special instruments and is time consuming. Second, the EIS measurement usually cannot be performed online.

Because many dynamic characteristics can be inferred from the continuous working charge/discharge sequence, in this paper, we utilize the continuous charge/discharge sequence of batteries when the battery is charged/discharged with a constant current for battery grouping. Actually, power batteries are usually processed with a formation procedure that often contains a number of alternate full charge and full discharge processes; these charge and discharge processes can be used for battery grouping.

Because the battery charge/discharge sequence is an ordered multidimensional data set, a charge/discharge sequence based battery grouping can actually be regarded as a time series clustering problem. There are two types of strategies for time series clustering. The first strategy is to extract some distinct features from the time series; the clustering can then be performed with this bag-of-features [12]. The second strategy is to utilize the whole sequence directly for clustering. At present, there are many time series clustering methods in the literature, including the classical K-means algorithm [13], the K-Nearest Neighbor (KNN) algorithm [14], etc. The K-means and KNN need to specify the number of clusters in advance, and when the number of clusters is unknown, it is necessary to use a certain method to estimate the number of clusters. Another similar problem for time series clustering is time series classification, which classifies time series to different classes and is a supervised learning problem, while the time series clustering problem is an unsupervised problem. Commonly used methods for time series classification are the shapelet based method [15], the recurrent neural network (RNN) based method [16], etc. In fact, sometimes we can use the time series classification method to accomplish time series clustering.

In this paper, we propose a battery grouping method according to the time series clustering methodology. The whole time series is utilized for clustering in this paper. The battery charge/discharge sequences are first denoised. The dynamic time warping (DTW) distances [17] between different battery charge/discharge sequences are then calculated, followed by a normalization step to form a similarity matrix, which is utilized by the affinity propagation (AP) algorithm [18] for clustering.

The paper is organized as follows. In Section 2, we define the battery grouping problem. In Section 3 we provide the framework of the proposed method and describe each part in detail. In Section 4, we show the experimental results. Finally, conclusions are provided in Section 5.

\section{Problem Formulation}

The purpose of battery grouping is to divide new batteries into different groups, with highest in-class characteristic similarities and lowest between-class characteristic similarities; this is a typical 
clustering problem. The only difference between the battery grouping problem and other common clustering problems is that the numbers of batteries in clusters for battery grouping problems are the same, but this is not a large problem. One can first cluster batteries with commonly used clustering algorithms, and then divide or merge clusters that have a different number of batteries other than the required number. This may not be an optimal solution, but it should be adequate. Therefore, in this paper, we regard the battery grouping problem as a common clustering problem.

As mentioned before, in this paper we use the full charge and discharge series for battery grouping. To obtain these charge and discharge series, we build an embedded system that can simultaneously measure the terminal voltages, temperatures and currents for 20 batteries, as shown in Figure 1.

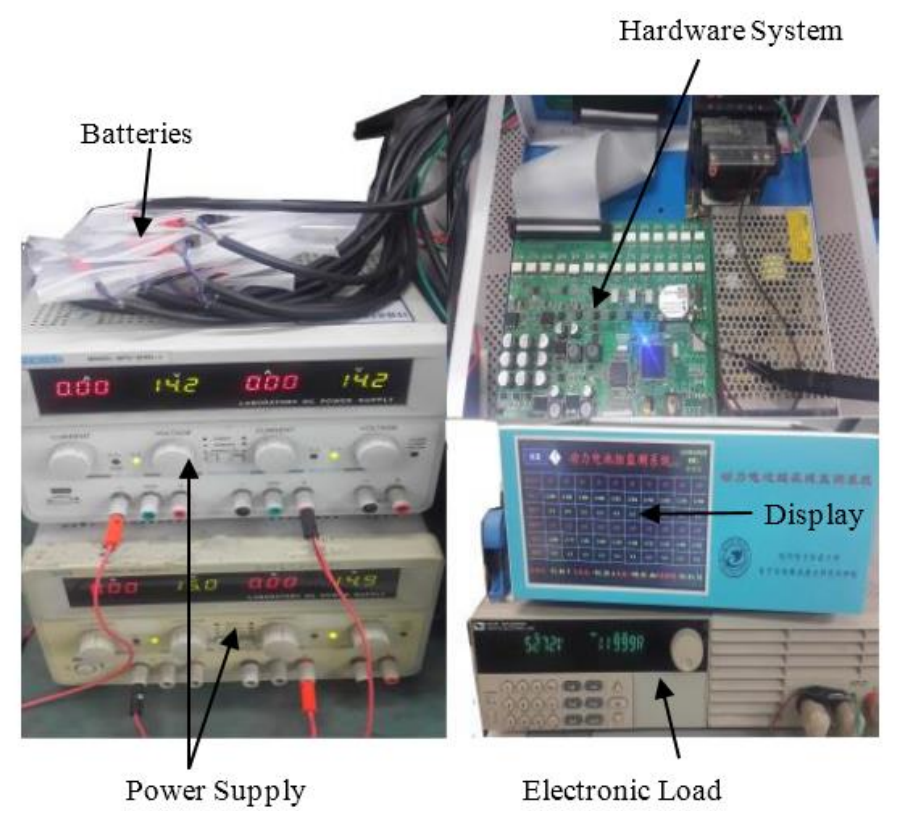

Figure 1. Our designed measurement system.

Typical battery discharge curves at a discharge rate of $0.6 \mathrm{C}$ for $\mathrm{LiMn}_{2} \mathrm{O}_{4}$ batteries are shown in Figure 2. At first glance, battery \#2 and \#4 are close to each other. Actually, the DTW distances, which will be further explained in the following section, between different sequences are as follows: $d_{12}=22.2572, d_{13}=4.6084, d_{14}=18.9139, d_{23}=11.8688, d_{24}=0.4057, d_{34}=8.5479$. A successful clustering algorithm should classify battery \#2 and battery \#4 in one group and battery \#1 should be classified to another group.

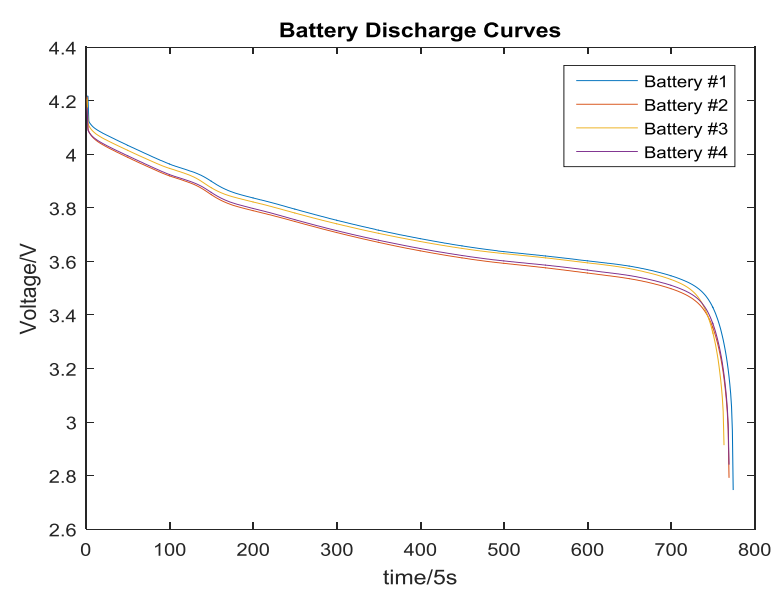

Figure 2. An illustration of the battery discharge curves. 


\section{AP Based Battery Grouping with the DTW Distance}

As mentioned before, we utilize the whole battery charge/discharge curve for battery grouping. For obtaining the battery charge curve, the battery is initially fully discharged, i.e., it has a state of charge of $0 \%$, the battery is then charged with a constant current until it is fully charged. During this process, the battery terminal voltages are measured and saved to form the battery charge curve. The process for obtaining the battery discharge curve is similar. Because both the charge curve and discharge curve are time-related, we regard battery grouping as a time series clustering problem. There are three important issues that should be considered before conducting time series clustering. First, noise may exist in the obtained battery charge/discharge data even if the battery is charged/discharged with a constant current. Second, the number of data points may be different with different batteries; thus the lengths of the time series may not be the same. Third, the number of clusters is unknown beforehand. To solve the first issue, a data denoising process should be used. In this paper, we utilize a wavelet based signal denoising [19] technology to smooth the battery charge/discharge data. Because the data lengths vary, the traditional distance metrics, such as the Euclidean distance, cannot be directly utilized. In this paper, we utilize the DTW distance to compute the similarities among different battery charge/discharge curves. Because the number of clusters is unknown, traditional data clustering methods, such as K-means and KNN cannot be directly utilized. For this reason, we use the AP algorithm to perform the time series clustering. The flow chart of the proposed method is shown in Figure 3.

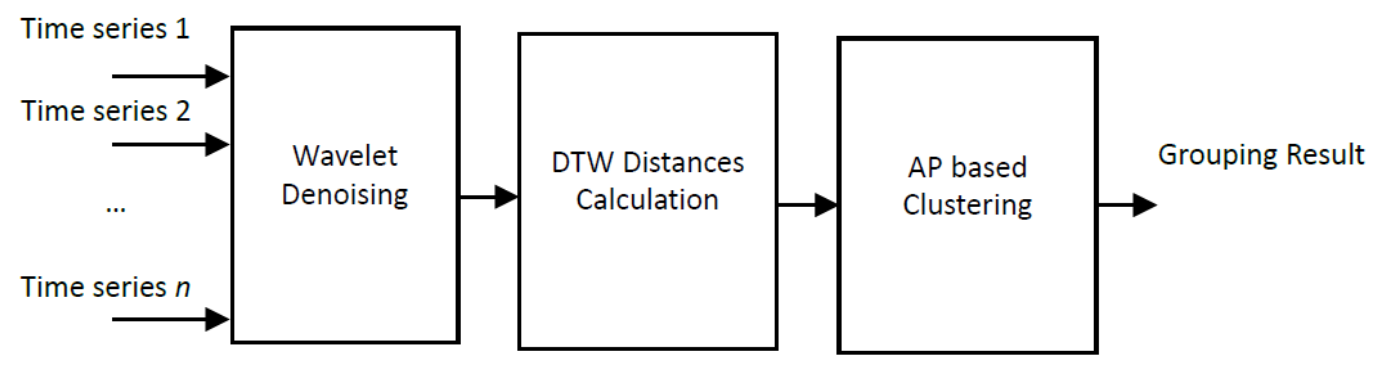

Figure 3. Flow chart of the proposed method.

\subsection{Wavelet Denoising}

Suppose the underlying model for the noisy signal $x(n)$ is basically of the following form:

$$
x(n)=s(n)+e(n)
$$

where $e(n)$ is Gaussian white noise with a zero mean and a standard variation of $\sigma$. The wavelet denoising mainly consists of the following three procedures:

Decomposition of the noisy signal $x(n)$ into $L$ levels and computation of the approximation coefficients of $x(n)$ at level $L$.

Denoising by thresholding the detail coefficients. In this step, a soft thresholding process is performed to the detail coefficients at levels 1 to $L$.

Reconstruction. Compute the reconstructed signal based on the original approximation coefficients of level $L$ and the thresholded detail coefficients of levels from 1 to $L$.

In this paper, we use the symlet wavelet "sym8" which is a compactly supported wavelet with the least asymmetry and the highest number of vanishing moments as the wavelet for signal decomposition. A total level of 5 is used. Figure 4 shows an example of wavelet denoising. As observed from Figure 3, the severe fluctuation of the original noisy signal has been successfully removed after denoising. 


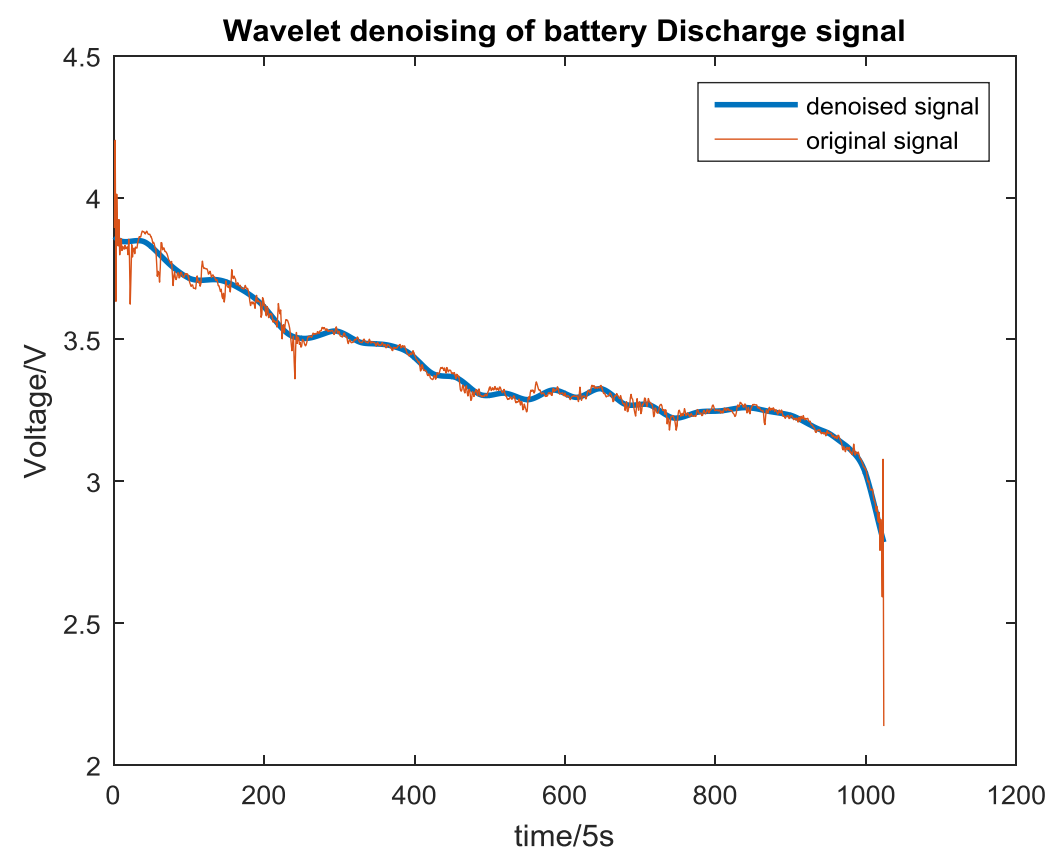

Figure 4. Wavelet denoising of time series.

\subsection{DTW Distance}

The similarity between two sequences can be measured by their distance. Suppose we have two sequences, $\mathbf{Q}=\left\{q_{1}, q_{2}, \ldots, q_{m}\right\}$ and $\mathbf{C}=\left\{c_{1}, c_{2}, \ldots, c_{n}\right\}$. There are several methods to compute the distance between $\mathbf{Q}$ and $\mathbf{C}$. For example, if $m=n$, we can use the Euclidean distance, i.e.,

$$
d=\sqrt{\sum_{i=1}^{m}\left|q_{i}-c_{i}\right|^{2}}
$$

If $m \neq n$, a simple way to calculate the distance is to rescale one of the sequences with interpolation so that the two sequences have the same length. Then, we can use the Euclidean distance to measure the similarity. However, this may not be a good strategy for time series with varying time and speed. As shown in Figure 5, two time series have similar shapes and the same length, but the feature points occur at different times. If we still use the Euclidean distance to measure the similarity between these two sequences, a large distance will be obtained, which may not be desired. For this situation, the DTW distance is a better choice to describe the similarity between them. In general, DTW is a method that calculates an optimal match between two sequences. The sequences are "time warped" non-linearly so that the key feature points can be properly aligned.

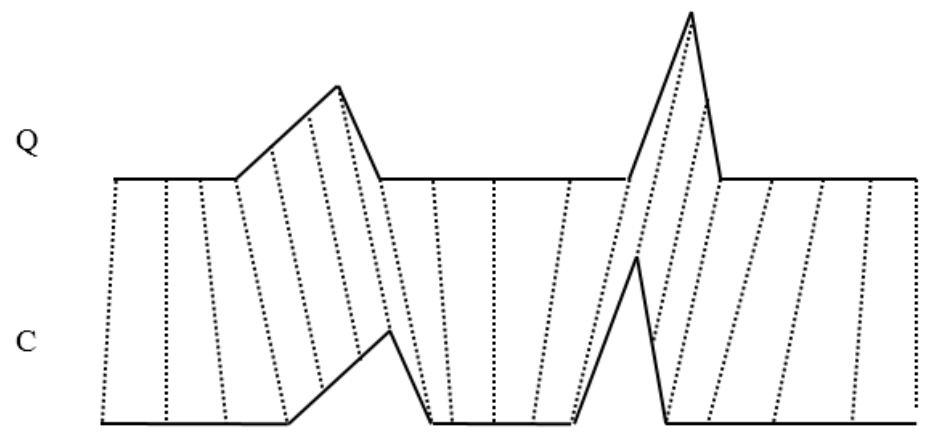

Figure 5. Feature points alignment in dynamic time warping (DTW). 
DTW utilizes dynamic programming to find the optimal mapping of points in two sequences and compute the distance between them. Detailed steps for computing the DTW distance can be found in several papers [20,21], and efficient algorithms can be found in [17].

Suppose we have $N$ sequences. After the computation of the DTW distances, we can obtain a distance matrix $\mathbf{D}=\left[d_{i j}\right]$, where $d_{i j}$ is the DTW distance between sequence $i$ and sequence $j$. Suppose the largest distance is $d_{\max }$ and the smallest distance is $d_{\min }$, we can then obtain the similarity matrix $\mathbf{S}=\left[s_{i j}\right]$, where $s_{i j}$ is the similarity of sequence $i$ and sequence $j$ and is computed as follows:

$$
s_{i j}=1-\left(d_{i j}-d_{\min }\right) /\left(d_{\max }-d_{\min }\right), i \neq j
$$

which is actually a normalization step.

According to the definition of distance, $d_{i i}$ is 0 . We define $s_{i i}$ as the mean similarity of the data points that connects to data point $i$, i.e.,

$$
s_{i i}=\frac{1}{N-1} \sum_{j=1, j \neq i}^{N} s_{i j}
$$

\subsection{AP Based Clustering}

$\mathrm{AP}$ is a clustering algorithm based on the concept of "message passing" between data points. Unlike traditional clustering algorithms such as K-means and KNN, AP does not require the number of clusters to be determined or estimated beforehand. At the beginning, all data points (a data point in this paper is actually a sequence) are regarded as exemplars. Each data point competes to become the cluster center with two types of message passing, i.e., the responsibility and the availability. This mechanism can avoid the influence of a random selection of initial cluster centers and the specified number of clusters. The responsibility $r_{i k}$ represents the message passed from data point $i$ to the candidate center $k$, which describes the appropriateness of data point $k$ to be the cluster center of data point $i$. However, the availability $a_{i k}$ represents the message passed from the candidate cluster center $k$ to data point $i$, which describes the appropriateness that data point $i$ chooses data point $k$ as its candidate center. The bigger the summation of $r_{i k}$ and $a_{i k}$, the more possible it is for data point $k$ to be a cluster center. $r_{i k}$ and $a_{i k}$ are initialized to be 0 at the beginning. After obtaining the similarity matrix $\mathbf{S}, r_{i k}$ and $a_{i k}$ are then computed as follows:

$$
\begin{gathered}
r_{i k}=s_{i k}-\max _{k^{\prime} \neq k}\left(a_{i, k^{\prime}}+s_{i, k^{\prime}}\right) \\
a_{i k}=\left\{\begin{array}{c}
\min \left\{0, r_{k k}+\sum_{i^{\prime} \notin\{i, k\}} \max \left(0, r_{i^{\prime}, k}\right)\right\}, i \neq k \\
\sum_{i^{\prime} \notin\{i, k\}} \max \left(0, r_{i^{\prime}, k}\right), i=k
\end{array}\right.
\end{gathered}
$$

The responsibility and the availability should then be updated with iterations as follows:

$$
\begin{aligned}
& r_{i k}=\lambda * r_{i k}^{\text {old }}+(1-\lambda) r_{i k} \\
& a_{i k}=\lambda * a_{i k}^{\text {old }}+(1-\lambda) a_{i k}
\end{aligned}
$$

where $\lambda$ is the damping coefficient, which has a value in the range of 0.5 to 1 . The iteration terminates when a maximum step is reached or the changes in values of $r_{i k}$ and $a_{i k}$ are not significant.

If for some battery $k$ we have $e_{k k}=r_{k k}+a_{k k}>0$, then battery $k$ is a cluster center, and we mark its corresponding element batteries.

More details on the AP algorithm can be found in [18].

\section{Experimental Results}

In this part, two types of experiments were performed. 
In the first experiment, the proposed battery grouping method was evaluated with real battery charge/discharge sequences. We obtained both the charge and discharge sequences for 94 new $\mathrm{LiMn}_{2} \mathrm{O}_{4}$ lithium-ion batteries with a nominal capacity of $2 \mathrm{Ah}$, though the proposed method can also be applied to other types of batteries. The charge curves were obtained when the batteries were charged with a constant current of $1.2 \mathrm{~A}$ until the charge cutoff voltage reached, and then charged under a constant voltage when the current dropped to $0.02 \mathrm{~A}$. The discharge curves were obtained when the batteries were discharged at a constant current of $1.2 \mathrm{~A}$. We then utilized the proposed AP based method for battery grouping. For a comparison, we also tested the K-means based spectral clustering method [22]. We used the silhouette index [23] to assess the performance of the methods.

The silhouette indexes, according to different numbers of clusters with different algorithms for the charge sequences, are given in Table 1 . According to the results in Table 1, for the charge curve, the best number of clusters for the AP algorithm and the spectral clustering algorithm are both 6 . The best silhouette index of the AP algorithm is larger than that of the spectral clustering algorithm. Figure 6 shows a visual display of the clustered similarity matrices when the charge curves are used for battery grouping. As observed from Figure 6, the clustered similarity matrix for the AP algorithm is much more diagonalized than that of the spectral clustering algorithm. Both Table 1 and Figure 6 show better performance with the proposed AP based algorithm.

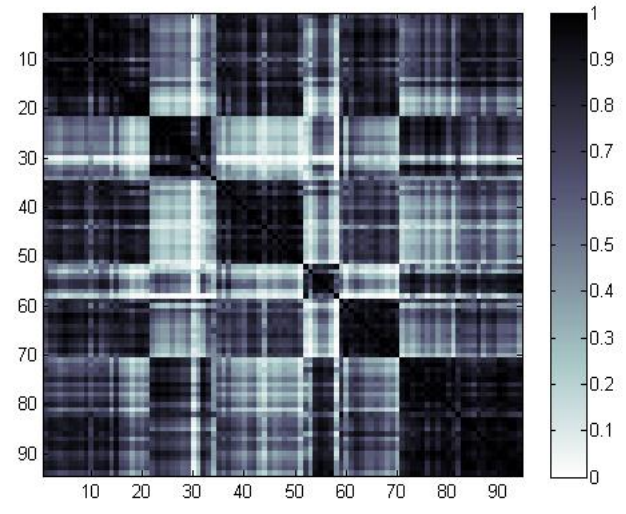

(a)

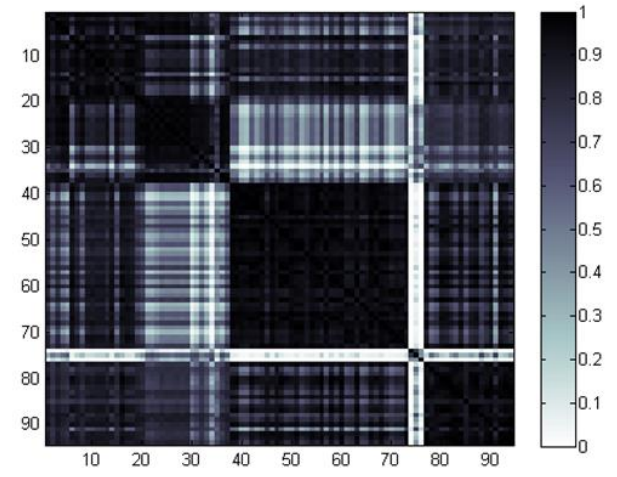

(b)

Figure 6. Clustered similarity matrices for the charge curves; (a) The AP algorithm; (b) The spectral clustering algorithm.

Table 1. Silhouette indexes for charge curves.

\begin{tabular}{ccc}
\hline \multirow{2}{*}{ Cluster Number } & \multicolumn{2}{c}{ Clustering Algorithm } \\
\cline { 2 - 3 } & AP & Spectral Clustering \\
\hline 6 & 0.9583 & 0.8452 \\
5 & 0.9225 & 0.7888 \\
4 & 0.8661 & 0.7603 \\
3 & 0.8298 & 0.7688 \\
2 & 0.7280 & 0.7773 \\
\hline
\end{tabular}

The clustering result for the charge curves based on the proposed AP based algorithm is shown in Figure 7, which also shows the effectiveness of the proposed algorithm. 


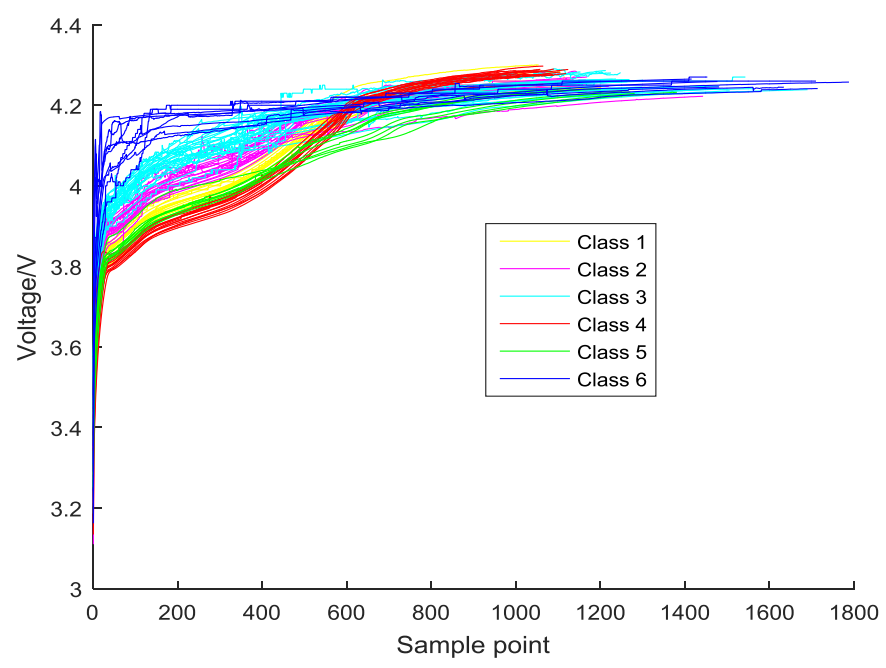

Figure 7. Clustering result for the charge curves with the proposed method.

The silhouette indexes, according to the different numbers of clusters with different algorithms for the discharge sequences, are given in Table 2. According to the results in Table 2, for the discharge curves, the best number of clusters for the AP algorithm is 5 , while the best number of clusters for the spectral clustering algorithm is 2 . The best silhouette index of the AP algorithm is still larger than that of the spectral clustering algorithm. Figure 8 shows a visual display of the clustered similarity matrices when the discharge curves are used for battery grouping. As observed from Figure 8, the clustered similarity matrix for the AP algorithm is much more diagonalized than that of the spectral clustering algorithm. Both Table 2 and Figure 8 also show better performance with the proposed AP based algorithm.

Table 2. Silhouette indexes for the discharge curves.

\begin{tabular}{ccc}
\hline \multirow{2}{*}{ Cluster Number } & \multicolumn{2}{c}{ Clustering Algorithm } \\
\cline { 2 - 3 } & AP & Spectral Clustering \\
\hline 5 & 0.9523 & 0.7501 \\
4 & 0.9139 & 0.5469 \\
3 & 0.8611 & 0.7169 \\
2 & 0.7583 & 0.9213 \\
\hline
\end{tabular}

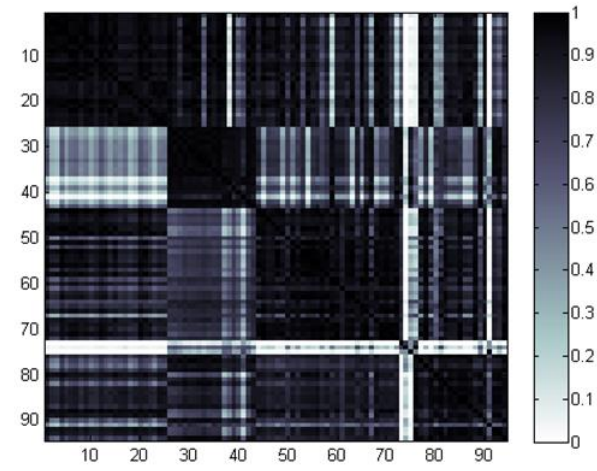

(a)

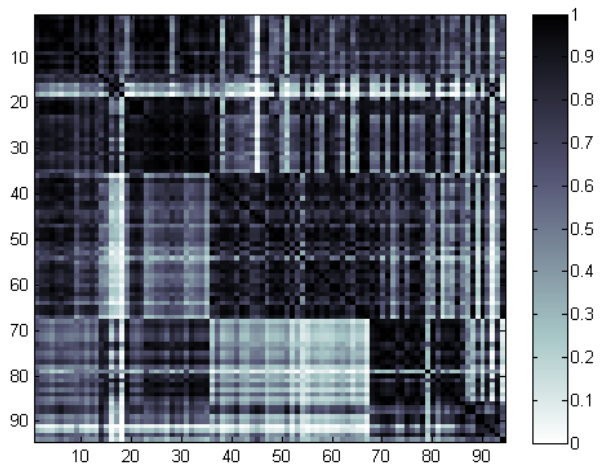

(b)

Figure 8. Clustered similarity matrices for discharge curves; (a) The AP algorithm; (b) The spectral clustering algorithm. 
The clustering result for the discharge curves based on the proposed AP based algorithm is shown in Figure 9, which also shows the effectiveness of the proposed algorithm.

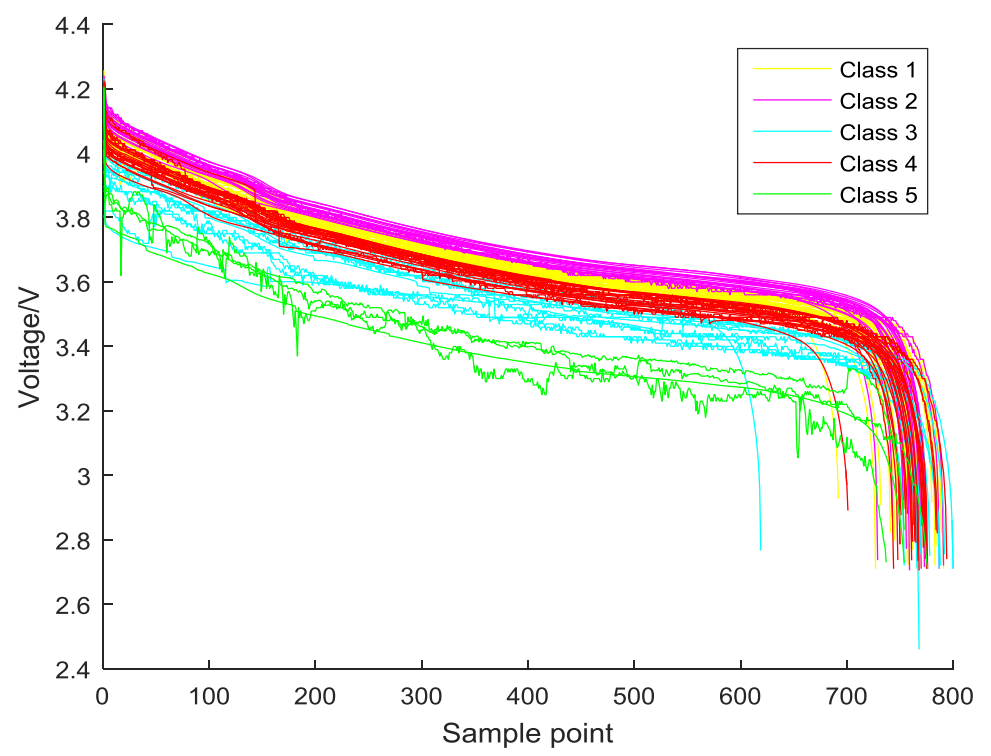

Figure 9. Clustering result for discharge curves with the proposed method.

In the second experiment, we built two battery packs, both containing four new battery cells. The four battery cells in the first battery pack were chosen from one of the clusters obtained according to the proposed algorithm. The battery cells in the second battery pack had similar discharge cutoff terminal voltages and charge static terminal voltages as utilized by many manufacturers. The two battery packs were then charged and discharged for many cycles under the same condition. Figure 10 shows the constant charge along with the voltage drop curve [24] at the 300th cycle for the battery cells in different packs. As observed from the figure, the characteristics of the battery cells in the first pack remain consistent, while the characteristics of battery cells in the second pack are inconsistent. This shows that battery grouping with the whole charge series is superior to that with only two distinct voltage values.

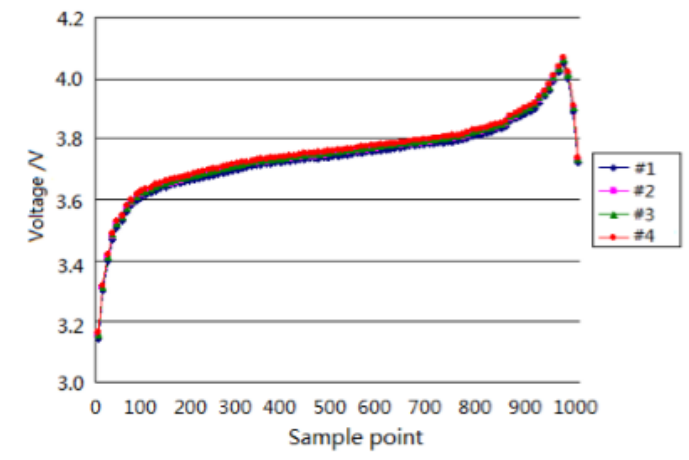

(a) Cells grouped with similar charge curves

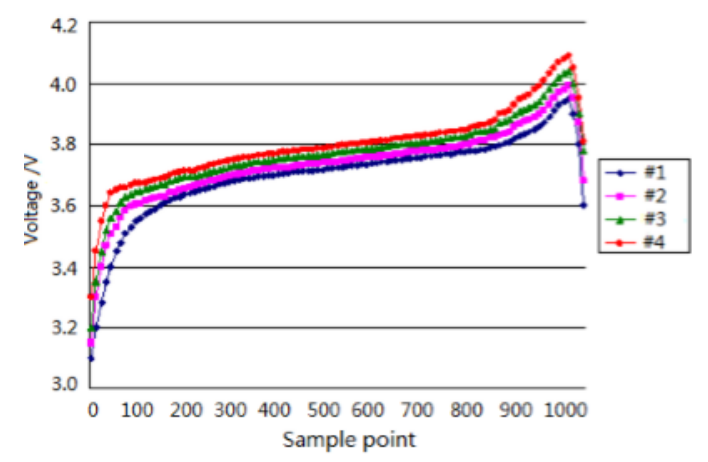

(b) Cells grouped with similar terminal

Figure 10. Constant charge along with the voltage drop characteristics of battery cells for two different battery packs; (a) cells grouped with similar charge curves; (b) cells grouped with similarterminal.

\section{Conclusions}

Battery grouping is a widely used technology to improve the performance of battery packs. In this paper, we propose a time series clustering based battery grouping method. The proposed method 
utilizes the whole battery charge/discharge sequence for battery grouping. The time sequences are first denoised with a wavelet denoising technique, the similarity matrix is then computed with the DTW distance, and finally, the time series are clustered with the AP algorithm according to the calculated similarity matrices. The silhouette index is utilized for assessing the performance of the proposed battery grouping method. Test results show that the proposed battery grouping method is effective.

In this paper, we performed a clustering based only on the charge or discharge time series of batteries, and an extension to clustering based on both charge and discharge time series is straight forward. However, from the results of Tables 1 and 2, different clusters are obtained for the same batteries when the charge and discharge time series are utilized. Therefore, research on a strategy to combine the charge and discharge time series is worthwhile. On the other hand, the time series used for battery grouping in this paper were obtained under constant charge/discharge rates $(0.6 \mathrm{C}$ for both charge and discharge). In the future, research on the influence of different charge/discharge rates on the performance of grouped battery packs may be valuable.

Acknowledgments: The authors would like to thank the Editor and the anonymous reviewers for their insightful comments and suggestions. This work was supported by Nature Science Foundation of China with Grant No. 61471151.

Author Contributions: Zhiwei He, Mingyu Gao and Guojin Ma conceived and designed the experiments; Lijun Tang performed the experiments; Zhiwei He and Yuanyuan Liu analyzed the data; Mingyu Gao and Yuanyuan Liu contributed analysis tools; Zhiwei He wrote the paper.

Conflicts of Interest: The authors declare no conflict of interest.

\section{References}

1. Wang, T.; Tseng, K.J.; Zhao, J.; Wei, Z. Thermal investigation of lithium-ion battery module with different cell arrangement structures and forced air-cooling strategies. Appl. Energy 2014, 134, 229-238. [CrossRef]

2. Xing, Y.; He, W.; Pecht, M.; Tsui, K.L. State of charge estimation of lithium-ion batteries using the open-circuit voltage at various ambient temperatures. Appl. Energy 2014, 113, 106-115. [CrossRef]

3. He, Z.; Gao, M.Y.; Wang, C.; Wang, L.; Liu, Y.Y. Adaptive state of charge estimation for Li-ion batteries based on an unscented Kalman filter with an enhanced battery model. Energies 2013, 6, 4134-4151. [CrossRef]

4. Gholizadeh, M.; Salmasi, F.R. Estimation of state of charge, unknown nonlinearities, and state of health of a lithium-ion battery based on a comprehensive unobservable model. IEEE Trans. Ind. Electron. 2014, 61, 1335-1344. [CrossRef]

5. He, Z.; Gao, M.; Ma, G.; Liu, Y.Y.; Chen, S.X. Online state-of-health estimation of lithium-ion batteries using Dynamic Bayesian Networks. J. Power Sources 2014, 267, 576-583. [CrossRef]

6. Gogoana, R.; Pinson, M.B.; Bazant, M.Z.; Sarma, S.E. Internal resistance matching for parallel-connected lithium-ion cells and impacts on battery pack cycle life. J. Power Sources 2014, 252, 8-13. [CrossRef]

7. Alaoui, C. Solid-state thermal management for lithium-ion EV batteries. IEEE Trans. Veh. Technol. 2013, 62, 98-107. [CrossRef]

8. Zeng, Y.; Yang, Y.X.; He, Z.W.; Gao, M.Y.; Wang, C.S.; Hong, M. Lead-acid Battery Automatic Grouping System Based on Graph Cuts. Electr. Power Compon. Syst. 2016, 44, 450-458. [CrossRef]

9. Seaman, A.; Dao, T.S.; McPhee, J. A survey of mathematics-based equivalent-circuit and electrochemical battery models for hybrid and electric vehicle simulation. J. Power Sources 2014, 256, 410-423. [CrossRef]

10. Mendoza-Hernandez, O.S.; Ishikawa, H.; Nishikawa, Y.; Marugama, Y.; Sone, Y.; Umeda, M. State of Charge Dependency of Graphitized-Carbon-Based Reactions in a Lithium-ion Secondary Cell Studied by Electrochemical Impedance Spectroscopy. Electrochim. Acta 2014, 131, 168-173. [CrossRef]

11. Galeotti, M.; Cinà, L.; Giammanco, C.; Cordiner, S.; Di Carlo, A. Performance analysis and SOH (state of health) evaluation of lithium polymer batteries through electrochemical impedance spectroscopy. Energy 2015, 89, 678-686. [CrossRef]

12. Baydogan, M.G.; Runger, G.; Tuv, E. A Bag-of-Features Framework to Classify Time Series. IEEE Trans. Pattern Anal. Mach. Intell. 2013, 35, 2796-2802. [CrossRef] [PubMed]

13. Aggarwal, C.C.; Reddy, C.K. Data Clustering: Algorithms and Applications; Chapman \& Hall/CRC Press: Boca Raton, FL, USA, 2013. 
14. Caraway, N.M.; McCreight, J.L.; Rajagopalan, B. Multisite stochastic weather generation using cluster analysis and k-nearest neighbor time series resampling. J. Hydrol. 2014, 508, 197-213. [CrossRef]

15. Zakaria, J.; Mueen, A.; Keogh, E. Clustering Time Series using Unsupervised-Shapelets. In Proceedings of the 2012 IEEE 12th International Conference on Data Mining (ICDM), Brussels, Belgium, 10 December 2012; pp. 785-794.

16. Najibi, E.; Rostami, H. SCESN, SPESN, SWESN: Three recurrent neural echo state networks with clustered reservoirs for prediction of nonlinear and chaotic time series. Appl. Intell. 2015, 43, 460-472. [CrossRef]

17. Begum, N.; Ulanova, L.; Wang, J.; Keogh, E. Accelerating Dynamic Time Warping Clustering with a Novel Admissible Pruning Strategy. In Proceedings of the 21th ACM SIGKDD International Conference on Knowledge Discovery and Data Mining, Sydney, Australia, 10 August 2015; pp. 49-58.

18. Frey, B.J.; Dueck, D. Clustering by passing messages between data points. Science 2007, 315, 972-976. [CrossRef] [PubMed]

19. Ding, Y.; Selesnick, I.W. Artifact-free wavelet denoising: Non-convex sparse regularization, convex optimization. IEEE Signal Process. Lett. 2015, 22, 1364-1368. [CrossRef]

20. Shokoohi-Yekta, M.; Wang, J.; Keogh, E. On the Non-Trivial Generalization of Dynamic Time Warping to the Multi-Dimensional Case. In Proceedings of the SIAM International Conference on Data Mining, Vancouver, BC, Canada, 30 April 2015.

21. Petitjean, F.; Forestier, G.; Webb, G.I.; Nicholson, A.E.; Chen, Y.; Keogh, E. Faster and more accurate classification of time series by exploiting a novel dynamic time warping averaging algorithm. Knowl. Inf. Syst. 2015, 47, 1-26. [CrossRef]

22. Dhillon, I.S.; Guan, Y.; Kulis, B. Kernel k-means: Spectral Clustering and Normalized Cuts. In Proceedings of the tenth ACM SIGKDD International Conference on Knowledge Discovery and Data Mining, Seattle, WA, USA, 22 August 2004; pp. 551-556.

23. Petrovic, S. A Comparison between the Silhouette Index and the Davies-Bouldin Index in Labelling Ids Clusters. In Proceedings of the 11th Nordic Workshop of Secure IT Systems, Linkoping, Sweden, 19 October 2006; pp. 53-64.

24. Lin, H.-T.; Liang, T.-J.; Chen, S.-M. Estimation of Battery State of Health Using Probabilistic Neural Network. IEEE Trans. Ind. Inform. 2013, 9, 679-685. [CrossRef]

(C) 2016 by the authors; licensee MDPI, Basel, Switzerland. This article is an open access article distributed under the terms and conditions of the Creative Commons Attribution (CC-BY) license (http://creativecommons.org/licenses/by/4.0/). 